\title{
LA HERENCIA SOCIAL Y MISIONOLÓGICA DEL PACTO EVANGÉLICO DE LAUSANA DE 1974
}

\author{
Juan Carlos Cárcamo² \\ Departamento de Teología \\ Universidad Evangélica de El Salvador \\ carcamoministerios@gmail.com \\ Recibido 17/04/16 \\ Aceptado 30/05/16
}

\section{Resumen}

En el trabajo se presentan los resultados de una investigación documental realizada para analizar la herencia social y misionológica contenida en el Pacto de Lausana, desarrollado como consecuencia del Encuentro Mundial de Evangelización que tuvo lugar en la ciudad de Lausana en el año de 1974; del mismo modo, se pretenden situar los aspectos más relevantes que permitan entender el origen de dicho Pacto y evaluar su pertinencia para el contexto salvadoreño. La investigación incluyó el análisis de los textos básicos generados en el Encuentro Mundial de Evangelización, primordialmente la declaración principal y los comentarios que realizara al mismo quien fuera el presidente del Comité Editor, el teólogo inglés John Stott. También se tomó en cuenta información documental secundaria que permitiera entender las motivaciones y el contexto del Pacto de Lausana. Entre los principales hallazgos encontrados se encuentra el hecho de que exista mucha literatura que comenta de manera negativa los eventos generados alrededor del Encuentro; pero, por otro lado, se puede afirmar también que existe una clara conexión entre lo que se denominó como el nuevo evangelicalismo derivado de Lausana con el movimiento latinoamericano conocido como Misión Integral. Se concluye que la herencia social del Pacto de Lausana es pertinente para el contexto evangélico salvadoreño, por lo cual debe promoverse su lectura y estudio para evitar una misión desarraigada de las necesidades humanas.

PALABRAS CLAVE: Pacto de Lausana; Misionología; Iglesias evangélicas; Misión integral; Evangelización; Tercer Mundo; pobreza

1 Mis más sinceros agradecimientos a la Escuela de Teología de la Universidad Evangélica de El Salvador por ofrecer su apoyo para la presente investigación de Catedra y al equipo del curso Practicas Ministeriales de la Licenciatura de Teología, conformado por su docente y el equipo de estudiantes por su interés en profundizar en esta temática.

2 Licenciado en Teología, Master en Investigación Científica por la Universidad Evangélica de El Salvador y egresado del Doctorado en Filosofía Iberoamericana de la Universidad Centroamericana José Simeón Cañas. 


\section{THE SOCIAL AND MISSIOLOGICAL LEGACY OF THE EVANGELICAL LAUSANNE COVENANT, 1974}

\section{Summary}

In the paper, the results of the documentary research are presented to analyze social and missiological heritage enclosed in the Lausanne Covenant, developed as a result in the World Meeting of Evangelization held in Lausanne in Year 1974; likewise, they are intended to place the most appropriate aspects that allow us understand the origin of the pact and assess their significance to the Salvadoran context. The research included an analysis of basic texts caused in the World Meeting Evangelization, primarily the main statement and comments made together by John Stott, the English theologian and also the chairman of the Editorial Committee. It was also taken into secondary documentary account information that there are attempts to understand the motivations and context of the Lausanne Covenant. Among the main findings is the fact that there are many negative literary commenting events generated around the meeting; but on the other hand, one can also say that there is a clear connection between what became known as the new evangelicalism (derived from Lausanne movement in Latin America) known as Integral Mission. It is concluded that the social heritage 'Pact Lausanne' is relevant to the Salvadoran evangelical context, which should endorse its reading and study to avoid human needs an itinerant mission.

KEYWORDS: Covenant of Lausanne; evangelical context; integral misión; poverty; El Salvador.

\section{INTRODUCCIÓN}

El paradigma misionológico más relevante de la última era, en el ámbito protestante y a nivel global, es el que se desprendió del Congreso Internacional de Evangelización Mundial en Lausana, Suiza, que se realizó del 16 al 21 de julio de 1974 (Stanley, 2013, 24).

El Congreso consistió en una reunión de alrededor 2,700 líderes protestantes de más de 150 países, sobre todo del denominado Tercer Mundo y con algunas de las ausencias más significativas, como fueron las de la delegación de China y Rusia. La revista Times se refirió al evento como la reunión de cristianos de la gama más amplia que se haya celebrado jamás. El lema del Congreso fue: "Que toda la tierra escuche su voz" (Bedrossian, 2014).

De este evento histórico se desprendió un documento que será conocido como el Pacto de Lausana. El documento tenía como propósito provocar o propiciar el nacimiento de algo nuevo en el mundo evangélico y no se quedara en una mera declaración de intenciones. Desde entonces, Lausana (1974) se reconoce, en la misionología protestante, como el inicio de un proceso, más que como un simple acontecimiento, porque daría origen a una serie de acciones integradas y orientadas a la movilización de la Iglesia protestante global desde un nuevo paradigma de 
misión (Comité de Lausana para la Evangelización mundial, 1984).

Los principios derivados de Lausana (1974) se difundieron por medio de otra serie encuentros posteriores entre teólogos protestantes, siendo los más representativos los de Manila (1989) y Ciudad del Cabo (2010) y los Encuentros CLADES (Encuentros Latinoamericanos de Evangelización) (Bonino, Sepulveda, \& Galvez, 2002).

El Congreso de Evangelización realizado en Manila en el año de 1989 reafirmó los compromisos suscritos en 1974 y se constituyó en un cónclave ecuménico relevante para dar seguimiento a la evangelización integral. De ese encuentro se desprendió una nueva declaración que contenía 21 premisas sobre la misionología protestante. Del mismo modo, el Congreso realizado en el año 2010 en Ciudad del Cabo mantuvo su relevancia como la expresión más incluyente del pensamiento evangélico global y ha marcado el rumbo de la misión de las iglesias evangélicas en los últimos 40 años, de acuerdo a representantes de la delegación salvadoreña en la más reciente edición de dicho Congreso. ${ }^{3}$

\section{METODOLOGÍA DE LA INVESTIGACIÓN}

La presente investigación es de índole documental e informativa, es decir, depende fundamentalmente de la información que se recoge o consulta en documentos, entendidos estos como todo material escrito que aporta información de

3 En la última reunión Global realizada en Ciudad El Cabo, Sudáfrica, hubo representación del Comité Lausana El Salvador (2011) y editaron un documento con sus valoraciones del mismo se tituló: "Compromisos de Cuidad del Cabo. Sus desafíos para la iglesia evangélica en El Salvador". Delegación salvadoreña en el Congreso Lausana II, Cuidad del Cabo, Sudáfrica. El prólogo fue escrito por el pastor general de la Iglesia ELIM, Mario Vega. un acontecimiento. Es informativa porque busca ofrecer una visión panorámica de la información más relevante vinculada con el Pacto de Lausana de 1974, sin tratar de aprobar u objetar alguna idea, sino solo analizar y seleccionar elementos relevantes (Cazares, 1990).

Para la presente investigación, se ha tenido acceso a documentos escritos que describen los hechos vinculados al Encuentro Mundial de Evangelización de 1974, especialmente, los documentos periódicos de Lausana $\mathrm{y}$, sobre todo, la edición del Pacto de Lausana expuesta y comentada por John Stott. También se han analizado numerosas fuentes secundarias que permiten entender el contexto en el que se desarrolló el Encuentro de Evangelización de 1974. Por último se ha hecho un análisis de la información de fuentes digitales, como el sitio web del Movimiento Lausana, del Joshua Project, entre otros.

Se debe aclarar que no se ha profundizado en los eventos posteriores al de 1974, a pesar de que estuvieron enmarcados en la iniciativa de Lausana y fueron organizados por el Movimiento Lausana, pero se decidió delimitar el presente análisis al contexto del evento que dio origen a todo el proyecto misionero, que luego sería conocido como nuevo evangelicalismo. Sin embargo, se hace una referencia a los Encuentros Mundiales de Evangelización realizados en Manila y Ciudad El Cabo, pero sin profundizar en el contexto o contenidos de dichos encuentros.

Para efectos de delimitación, la presente investigación documental se centra en los temas sociales recogidos en el Pacto de Lausana de 1974, sin profundizar en otros aspectos sobresalientes de dicho documento. 


\section{ANÁLISIS Y DISCUSIÓN DE RESULTADOS}

\section{Antecedentes del Congreso Mundial de Evangelización de Lausana de 1974}

La Conferencia Mundial Misionera de Edimburgo, realizada en Escocia en 1910, es el antecedente inmediato para establecer el avance del protestantismo mundial en el siglo XX; sin embargo, en este Congreso no estuvo presente la región latinoamericana. Baez-Camargo lo interpretó como una señal de hegemonía por parte del paternalismo Victoriano. En reacción a lo ocurrido en Edimburgo, se convocó al Congreso Misionero de Panamá, en 1916, por parte de líderes y misioneros norteamericanos y europeos que no compartían el anterior criterio de hegemonía transcultural.

Las características de la misionología derivada de ese periodo fue el de una teología elaborada desde los países misioneros, con un énfasis biblicista $y$ pietista, enfocada en repetir formulas centradas en la práctica homilética y en la propaganda. También estaba caracterizada por una relación con el liberalismo político latinoamericano y revestido de un tono y un estilo extranjerizantes.

Es en ese marco que la Asociación Evangelística Billy Graham consideró clave dar continuidad al Congreso Mundial de Evangelismo que se había realizado en Berlín, Alemania, en 1966; y para ello, convocaron a otras reuniones como la de 1970 y luego también en 1971, asimismo realizadas en Berlín, con un grupo pequeño de 16 líderes protestantes y asesores para revisar los principios derivados del Congreso de Berlín. Este proceso fue el que sirvió para la gestación del Congreso Mundial de Lausana, realizado en Suiza en 1974. Otro afluente que contribuyó a preparar el escenario en la conciencia de los protestantes, previo al Congreso de Lausana, fue la Asamblea del Consejo Mundial de Iglesias realizada en Lausana en 1968, la cual tuvo una fuerte connotación ecuménica. Es en esta Asamblea donde se define la misión de la Iglesia en términos de humanización y liberación política. Por lo anterior, la Fundación Graham consideró la necesidad de convocar a un evento en el que el liderazgo participante fuera eminentemente evangélico. Por otro lado, los líderes emergentes del movimiento estudiantil evangélico en América Latina dieron alta prioridad a la construcción de un pensamiento bíblico que respondiera al análisis marxista de la injusticia social en la sociedad. A través del International Fellowship of Evangelical Students (IFES) en América Latina, se dieron pasos importantes hacia un nuevo estilo de ser evangélico mediante la inmersión en procesos de reflexión orientados hacia un criticismo social y a una inusual conciencia del daño potencial de un imperialismo religioso (Stanley, 2013, 3 y 5).

Figura clave en la organización del Congreso de Lausana fue Charles Troutman, miembro del Latin American Mission y líder en el American Inter-Varsity, debido a su rol en la conformación de la Comunidad Latinoamericana de Ministerios Evangélicos (CLAME). Troutman fue quien propuso la incorporación de líderes cristianos jóvenes de América Latina e incorporar la visión del denominado Tercer Mundo. Es en este marco que se eligen como representantes del liderazgo juvenil cristiano latinoamericano a Samuel Escobar, Rene Padilla, Sergio García y a Orlando Costas. El Comité a cargo de escribir el Pacto estuvo conformado por John Stott, como encargado, Hudson Armerding (Presidente del Wheaton College), Samuel Escobar, J. D. Douglas y Leighton Ford. 
Algunos sectores de las nuevas iglesias evangélicas latinoamericanas conservadoras y algunas de las más radicales iglesias evangélicas del Norte, como la Iglesia Menonita Americana, presentaron lo que llamaron una respuesta a Lausana, según la cual cualquier intento por conciliar el evangelismo con la acción social como demoníaco.

El Primer Congreso Mundial de Evangelización (1974) ocurrió a una década de distancia del Concilio Vaticano II, realizado entre los años 1962 y 1965, mismo que traería importantes cambios en los paradigmas misionológicos de la Iglesia católica. Uno de los componentes fundamentales derivados del Concilio Vaticano II fue la opción preferencial por los pobres (Guerrero, 2010, 5).

A inicios de los años 1970 se comenzaron a manifestar señales de un despertar en la conciencia social en el evangelicalismo más conservador de occidente. Algunos de los elementos que inspiraron esta reacción fueron el redescubrimiento de la tradición anabaptista y su discipulado radical, así como el involucramiento de algunos líderes protestantes negros en el movimiento por los derechos civiles en los Estados Unidos (Stanley, 2013, 2).

El Comité Organizador de Lausana estuvo conformado por 31 miembros, de los cuales 12 eran de los Estados Unidos, 6 asiáticos y 2 africanos, y el resto de diferentes lugares del mundo. El Comité estaba completamente conformado por hombres. Uno de los invitados de Graham fue el clérigo anglicano John Stott, quien no pareció interesado inicialmente en el asunto, y más bien manifestó a Graham su deseo de ver claramente el propósito de la reunión en términos de la formulación de una estrategia detallada para la evangelización mundial. Debido a una presión ejercida especialmente por agencias misioneras y líderes de Gran Bretaña, la Asociación Evangelística Billy Graham retiró todos sus representantes del Comité Organizador, a excepción de Leighton Ford. Esa presión se mantuvo en los meses anteriores a la realización del evento, especialmente por la percepción de ser un evento con una clara influencia estadounidense. La participación se definió con base a un criterio de 7 participantes por cada millón de protestantes en la población del país, pero posteriormente el criterio se cambió a 2 participantes por cada 10 millones de personas no evangelizadas en el país (Stanley, 2013, 7 y 9).

La participación de los invitados latinoamericanos en el Congreso de Lausana de 1974 cobraría una enorme relevancia por sus posicionamientos respecto de la necesidad de una nueva comprensión de los paradigmas misioneros. Por ejemplo, Rene Padilla introdujo el debate respecto del evangelismo y el mundo, señalando la dificultad de preservar una cultura cristiana como una variante de la cultura americana, incluyendo muchas de sus estrategias de evangelización. Del mismo modo, la ponencia desarrollada por Samuel Escobar sobre el evangelismo y la búsqueda de libertad, justicia y plenitud para los hombres, señalaba el riesgo de hacer del cristianismo una expresión de la ideología de occidente. Por su parte, Orlando Costas también desarrolló el tema de evangelismo profundo, en el que planteaba una evangelización radical y contextual, aplicando principios del pensamiento de Paulo Freire, como el de concientización, para referirse a la aplicación del evangelio a todas las áreas de la vida del ser humano. Se afirma que hubo intentos al interior del Congreso Mundial de Evangelización de Lausana de 1974 por conformar un 
Consejo Mundial de Iglesias Evangélicas que hiciera contrapeso al ya constituido Consejo Mundial de Iglesias, pero algunos de los participantes consideraron sería un hecho desastroso (Stanley, 2013, 13-14).

Otro de los intentos gestados al interior del Congreso de Lausana fue evitar una moratoria generalizada de las iniciativas de evangelización mundial, tal y como proponían algunos representantes. Para entender mejor lo señalado en el párrafo anterior, se debe recurrir a otra figura relevante de dicho proceso suscitado alrededor del Congreso de Lausana. Esa figura fue Gatu. El líder evangélico africano levantó la discusión acerca de la moratoria para el envío de misioneros del Norte a los países del Sur para evitar la dependencia local de la ayuda externa. Esta discusión fue reiterada por Gatu en la conferencia del Concilio Mundial de Iglesias, y su Comisión de Misiones Mundiales y Evangelismo realizada en Bangkok en enero de 1973; y reiteró el mismo llamado en la Conferencia Africana de Iglesias en Lusaka en mayo de 1974, de manera que se convirtió en un tema inevitable en Lausana, realizado solo dos meses después, en julio de 1974. Fue en esta última reunión en la que se desarrolló un encuentro privado entre Gatu, Graham, Stott y Michael Green, en la que suscribieron lo que se denominó el Nuevo Evangelicalismo, como una expresión más sustentada desde el Sur (Stanley, 2013, 15).

Si en los preparativos del Congreso Mundial de Evangelización de Lausana, la figura predominante fue la de Billy Graham, al final del evento la figura más notable fue la de John Stott. Incluso, la Iglesia Anglicana consideró el evento como un triunfo para el anglicanismo, entendido como una alternativa intermedia entre el fundamentalismo americano y el nuevo radicalismo evangélico del Sur.
Pero también se levantaron voces contrarias al espíritu de Lausana: las más sobresalientes fueron las que consideraron que algunos grupos radicales del sur habían sido insensibles a la necesidad de la proclamación del evangelio. También manifestaron su inconformidad por el aparente ataque realizado por teólogos latinoamericanos, como el que se le atribuyó a Samuel Escobar, y por su consideración del imperialismo americano o la cultura cristiana, señalada por Padilla. Uno de los autores más representativos de estos señalamientos fue Harold Lindsell editor de la revista Cristianity Today (Coeller, 2012). Otros detractores consideraron que en Lausana hubo intentos por confundir evangelismo con acción social, confundir evangelismo con cooperación cristiana y confundir evangelismo con nutrición cristiana. Peter Wagner, del Fuller Theological Seminary School of World Mission fue uno de los promotores de esta posición en contra de los principios derivados del Pacto de Lausana (Stanley, 2013, 21 y 23).

\section{Los principios sociales contenidos en el Pacto de Lausana sobre misionología}

En la declaración de 1974 pueden encontrarse al menos 15 temas claves que conforman el Pacto de Lausana, entre los que destacan: el propósito de Dios, la autoridad y el poder de la Biblia, la singularidad y la universalidad de Cristo, la naturaleza de la evangelización, la responsabilidad social de los cristianos, la iglesia y la evangelización, la cooperación en la evangelización, las iglesias en coparticipación evangelística, la urgencia de la tarea evangelística, la evangelización y la cultura, la educación y el liderazgo, el conflicto espiritual, la libertad y la persecución, el poder del Espíritu Santo y el regreso de Cristo. El espíritu originario 
del Pacto de Lausana estaba orientado hacia una clara integralidad, como por ejemplo cuando se afirmaba la siguiente declaración: “...debemos compartir su interés (el de Dios) por la justicia y la reconciliación en toda la sociedad humana, y su interés por la liberación de los hombres de toda clase de opresión". La anterior afirmación deja entrever un sólido compromiso de la misionología evangélica derivada de Lausana con una transformación integral de la persona humana (Comité de Lausana para la Evangelización Mundial, 1984, 4 y 22).

Acerca de la actividad socio histórica del creyente, Lausana situaba un principio que rompía con cualquier dicotomía teológica al decir: "Afirmamos... que tanto la evangelización como el involucramiento socio-político forman parte de nuestro deber cristiano. Porque ambos son expresiones necesarias de nuestras doctrinas sobre Dios y sobre el hombre, sobre nuestro amor al prójimo y sobre nuestra obediencia a Jesucristo". De manera categórica, la Declaración señalaba la necesidad de hacer incidencia cristiana en situaciones que atentan contra la dignidad humana y van en contra de la ética derivada de las Escrituras, al señalar: "El mensaje de salvación, implica también un mensaje de juicio sobre toda forma de alienación, opresión y discriminación, y no debemos tener miedo de denunciar el mal y las injusticias donde quiera que existan.... Lo anterior supone un modelo de testimonio de mayor profundidad ética (Comité de Lausana para la Evangelización Mundial, 1984, 22).

En su comentario sobre la Declaración de Lausana, Jonh Stott indica que un grupo numeroso de participantes del Congreso de 1974, respecto de la afirmación anterior y queriendo enfatizar aún más en la radicalidad en cuanto al discipulado, deseaban redactar el anterior párrafo del modo siguiente: "Debemos repudiar como diabólico el intento de abrir un abismo entre la evangelización y la acción social". Lo mencionado sugiere que la generación participante del Congreso de 1974 concibió una enorme necesidad de comprometer la evangelización con la acción social, sobretodo la que transforma las condiciones de vida de las personas como expresión del Reino de Dios (Comité de Lausana para la Evangelización Mundial, 1984, 22).

Respecto de la tarea evangelística, Lausana planteaba algunos escenarios misioneros muy concretos, como por ejemplo el siguiente: "A todos nos sacude la pobreza de millones de personas, y nos molestan las injusticias que la causan. Aquellos de nosotros que vivimos en circunstancias holgadas aceptamos nuestro deber de desarrollar un estilo de vida sencillo a fin de contribuir más generosamente, tanto a la ayuda material como a la evangelización". Stott señala que tanto esta afirmación, como el llamado a tener un estilo de vida sencillo, provocó ansiedad en algunos de los futuros firmantes, y que incluso algunos preferían incorporar un adverbio de cantidad para efectuar un ejercicio de comparación que permitiera hacer más viable este postulado (Comité de Lausana para la Evangelización Mundial, 1984, 32 y 37).

Del mismo modo, la declaración, al referirse al ámbito de la cultura, establecía lo siguiente: "El evangelio no presupone la superioridad de ninguna cultura sobre otra... con demasiada frecuencia las misiones han exportado juntamente con el Evangelio a una cultura ajena, y, a veces, las iglesias han estado atadas a la cultura en vez de estarlo a las Escrituras" (Comité de Lausana para la Evangelización Mundial, 1984, 38). Este último aspecto se vuelve relevante en cuanto a la discusión 
sobre abandonar los viejos postulados colonizadores, dejar a un lado cualquier pretensión de conquista y encarnarse en las realidades de los pueblos receptores.

\section{Contexto históricos del Congreso de Evangelización Mundial de Lausana, 1974}

\section{a) Una teología misionera antes de Lausana}

Como señala Arturo Piedra, las primeras décadas del protestantismo en América Latina crearon desconfianza entre la población del Continente debido a ciertas acciones y percepciones como las siguientes: las iglesias protestantes eran vistas como filibusteras, sus vínculos con empresas comerciales y el apoyo a acciones de los Estados Unidos. Además de todo esto, la promoción que realizaron los movimientos protestantes del panamericanismo y la noción de un protestantismo como religión del progreso, contribuyó a una expansión lenta de las misiones protestantes (Piedra, A. 2002, 18).

Como explica Sosa Siliezar, el "protestantismo liberal" es una categoría utilizada para aquellos líderes que dejaron ver su simpatía por el proyecto político liberal como esperanza para el futuro de América Latina. Este movimiento fue clave para el surgimiento de una interpretación teológica desde la periferia del poder protestante global. El Comité de Cooperación para América Latina (1913), el Congreso de Obra Cristiana (Panamá, 1916), los congresos evangélicos de Montevideo (1925) y La Habana (1929), y la Primera Conferencia Evangélica en América Latina (1949) destacaron en dicho movimiento (Sosa, 2007, 47).

Pero el verdadero cambio de actitud de los evangélicos hacia la responsabilidad social va a comenzar a generar declaraciones como la de Wheaton, Illinois de 1966, (Padilla, 1985). Emilio Castro y UNELAM. (Dussel, 1992) van a ser claves en la concepción de la evangelización y las misiones, y ya para 1974, varios teólogos latinoamericanos, como Samuel Escobar, señalaban las condiciones imperantes de injusticia en el ámbito de América Latina. En este periodo se crea la Comisión de Iglesia y Sociedad en América Latina.

En el ámbito socio político, la Guerra Fría entre Estados Unidos y la Unión Soviética, el conflicto en Vietnam y la presencia de las tropas estadounidenses, la inestabilidad política estadounidense debido al escándalo Watergate, la Revolución chilena, el internacionalismo cubano que había expandido su presencia a territorios africanos como Angola, Namibia y Sudáfrica en búsqueda de la descolonización fueron algunos de los acontecimientos que sirvieron de marco contextual para el Congreso de Lausana de 1974 (Guerrero, 2010, 8).

\section{b) Expresiones de una teología misio- nera después de Lausana}

Inmediatamente después de Lausana, se convocó a una reunión en Pattaya, Tailandia (1980) por parte del Comité de Evangelización Mundial. Esta consulta fue considerada una reacción contra Lausana y su progreso en la formación de una misión integral. La reunión de Pattaya retomó el lema de Lausana: "Que todo escuche", pero agregó una especie de aclaratoria diciendo: "Alcanzando los no-alcanzados". Dicho Congreso se enfocó en la metodología de la misión. El centro de esta conferencia fue la preocupación por el "principio de la unidad homogénea” y cómo podemos evangelizar al mundo a través de "grupos metas". Entre los diecisiete grupos blancos/meta mencionados estaban los musulmanes, los pobres urbanos, los adherentes a los 
nuevos movimientos religiosos y cristianos nominales entre los católicos romanos (Dayton, 1980).

La heredera de Lausana fue la teología de la Misión Integral, la cual fue impulsada desde América Latina. René Padilla considera que la Misión Integral es la misión orientada a la satisfacción de las necesidades básicas del ser humano, incluyendo su necesidad de Dios, pero también su necesidad de amor, alimento, techo, abrigo, salud física y mental y sentido de dignidad humana (Padilla, 2006, 11).

Se han utilizado diferentes términos y diferentes connotaciones para referirse a la Misión Integral. Harold Segura explica que se ha hablado de Misión Holística para referirse también a ese esfuerzo de reflexión teológica que fue adquiriendo la fuerza de un movimiento regional y global de compromiso social para la iglesia, sobretodo, evangélica (Segura, 2006).

La declaración del Segundo Congreso de Evangelización realizado en Manila en 1989 adoptó un tono menos comprometido con el ámbito social y se limitó a hacer algunas referencias a problemáticas estructurales como la pobreza y la corrupción política. A pesardeloanterior, sedestacanafirmaciones como las siguientes: "Nos arrepentimos de haber sido indiferentes al clamor del pobre y por haber mostrado preferencia por el rico". Esta sería una de las referencias contenidas en la declaratoria de Manila sobre las problemáticas sociales, sin que profundice con la intencionalidad que había tenido el encuentro de 1974. A pesar de lo anterior, el valor misionero de Manila estuvo en el hecho de haber dado origen a más de 300 asociaciones estratégicas para la evangelización mundial, es decir, su aporte fue en términos de práctica misionera (Movimiento de Lausana, 2016)
En el marco del Tercer Congreso Misionero realizado en Ciudad del Cabo Sudáfrica en 2010, se redactaron los documentos del Cabo en los cuales el tema estructural de la realidad y de las problemáticas humanas volvió a cobrar relevancia en el ámbito misionológico de la Iglesia evangélica global. Por ejemplo, la siguiente afirmación establece una posición acerca de las problemáticas recientes relacionadas con la práctica misionera evangélica y la dignidad de las personas: "Cuando la enseñanza de la prosperidad se da en el contexto de la pobreza, debemos contraponerle una compasión auténtica y acciones que traigan justicia y una transformación duradera a los pobres" (Movimiento de Lausana).

Al menos se hacen 12 afirmaciones en los documentos de Ciudad del Cabo a aspectos vinculados con la pobreza y a las personas que viven en condiciones de exclusión. La siguiente afirmación refleja ese espíritu de compromiso: "Este amor por los pobres exige que no sólo amemos la misericordia y las acciones de compasión, sino que también hagamos justicia denunciando y oponiéndonos a todo lo que oprime y explota a los pobres". Y si bien se reconoce el papel de la Iglesia como agente de transformación, no se alcanza el nivel de aspiración que caracterizó al primer Congreso de Evangelización realizado en Lausana (Comité Lausana El Salvador, 2011).

En el marco de las organizaciones paraeclesiásticas, en el año 2001, alrededor de 140 practicantes de proyectos sociales, líderes de iglesias y teólogos se juntaron en Oxford, Inglaterra, para discutir acerca de la misión holística y el término que decidieron adoptar fue el de Misión Integral. Esta reunión lanzó la Red Miqueas, en la cual se aprobó una declaración sobre la Misión Integral (Carlisle, 2002, 19). 


\section{CONCLUSIONES Y RECOMENDACIONES}

La misión puede tener diferentes modelos para explicarse e implementarse. Algunos autores identifican 3 tradiciones como principales: la misión como descubrimiento de la verdad, misión como salvación de almas y crecimiento de la Iglesia y misión como transformación. Se recomienda profundizar en autores como Bevans y Schroeder quienes han delineado con claridad estas tres corrientes (Bevans \& Schroeder, 2009).

El modelo societario tuvo gran influencia entre protestantes, sobre todo a partir de la Conferencia Misionera de Edimburgo. Así también, en términos de teología de la misión, diferentes documentos definen los elementos fundamentales. ${ }^{4}$ Se debe destacar, además, que en la historia de los movimientos misioneros cristianos, al menos en América Latina, la misión, tanto evangélica como católica, ha estado permeada por un fuerte sentido de dominación y su interpretación ha sido dada en clave de poder, tal y como han demostrado historiadores como Jean Pierre Bastián, entre otros (Bastián, 2006).

En ese sentido, el Pacto de Lausana de 1974 viene a ser un instrumento misionero fundamental para la Iglesia evangélica a nivel global. El contexto en que surge y los resultados del Encuentro Mundial de Evangelización de Lausana reflejan una tensión continua entre diferentes cosmovisiones acerca de la

4 Se recomienda revisar documentos como el decreto conciliar Ad Agentes, para el caso de las iglesias ortodoxas en el cual se desarrolló la idea de la Missio dei, el Evangelii nuntiandi vinculado al protestantismo conciliar, el Redemptoris misio, clave para la compresión de los postulados más evangélicos y sobretodo pentecostales a partir de la misión como anuncio de Jesucristo, Salvador Universal. manera de ejercer la vida cristiana y cómo promoverla a nivel global. Diferentes fuentes reflejan un entramado complejo de intereses culturales, sociales, políticos y hasta económicos en la agenda misioneras evangélicas y protestantes a nivel global. Se recomienda a futuros investigadores ir más a fondo para situar más claramente los intereses que llevaron a cambios radicales entre el espíritu de Lausana de 1974 y otros encuentros posteriores, como el de Manila 1989.

Un análisis eminentemente instrumental se limitaría a la afirmación de que la presencia cristiana en el Continente es significativa a partir de los datos censales y de los informes que muestran que, tanto católicos y protestantes, tienen una presencia históricamente relevante en la vida de los pueblos y en su cohesión social. Al menos eso es lo que indican organismos como el Joshua Project (Joshua Project, 2015).

Si lo único importante a destacar de la misión cristiana se redujera al conteo de número de adeptos, cantidad de iglesias en funcionamiento, líderes entrenados en el manejo de lo religioso o si el conjunto de categorías religiosas contenidas en el cristianismo tienen un sentido eminentemente religioso y no están llamadas a ofrecer ningún valor práxico, entonces, el cristianismo vendría a ser un mecanismo a implementarse solamente al interior de un espacio social denominado iglesia, pero carecería de validez para permear la vida de los pueblos, es decir, transformar la realidad histórica como demandan autores como Ignacio Ellacuría (Ellacuría, 2009, 281).

No existen suficientes evidencias si alguna institución o iglesia adoptó de forma íntegra los principios del Pacto de Lausana en su práctica de Ministerio, sin embargo existen algunas publicaciones escasas que dan 
testimonio de que algunos ministerios y, sobre todo, organismos para-eclesiásticos sí han estado vinculados con los principios derivados del Congreso de Lausana en sus diferentes ediciones (Comité Lausana, El Salvador, 2011).

Los principios del Pacto de Lausana pueden ser de absoluta aplicabilidad en el contexto salvadoreño, pero para lograrlo será necesario darlos a conocer, máxime frente a al auge de una misionología empírica y asistémica que produce cifras de adeptos pero no transformación social.

La idea de contar con algunos principios rectores que sirvan para orientar las diferentes acciones dentro de la diversidad del movimiento protestante salvadoreño puede resultar utópico, al mismo tiempo que pertinente, debido a la dispersión que prevalece en el espectro de la Iglesia evangélica o protestante y por la manera en que ello afecta el impacto en cuanto a incidencia en temas de índole social, como la búsqueda de la justicia, la erradicación de la pobreza y el combate a la corrupción.

Promover un pacto misionológico puede ser oportuno si cumple algunas condiciones como las siguientes: 1. Que ofrezca apertura ecuménica, es decir, que sea lo suficientemente flexible para respetar la diversidad, pero lo suficientemente claro para cohesionar a diferentes expresiones de un mismo espectro. 2. Que sea operacionalmente eficiente en su implementación para que sea sostenible y medible en cuanto a sus resultados. 3. Que su fundamentación sea la praxis, y desde la periferia, de modo que no se convierte en un mero ejercicio de hegemonía transcultural. 4. Que sea expresión del Reino de Dios y que por tanto dé prioridad a la persona humana por sobre los procesos y los fines. 5. Que sea exegéticamente profundo para revelar lo histórico del mensaje del evangelio y su pertinencia para nuestra realidad histórica.

\section{FUENTES CONSULTADAS}

Bastián, J. P. (2006). “De los protestantismos históricos a los pentecostalismos latinoamericanos: Análisis de una mutación religiosa". Revista de Ciencias Sociales (Cl), núm. 16, 38-54. Universidad Arturo Prat Tarapacá, Chile.

Bedrossian, R. (2014). Cómo pensar la misión de la iglesia contemporánea, a los 40 años del Pacto de Lausana, en el contexto urbano de las ciudades latinoamericanas. Tesis presentada para optar al título de Master en Teología por el South African Theological Seminary.

Bonino, M, Sepulveda J, \& Galvez R. (2002). Unidad $y$ diversidad del protestantismo latinoamericano, el testimonio hacia el tercer milenio: Palabra, Espíritu y Misión. 1a. Ed, Buenos Aires: Kairos.

Cazares, L. (1990). Técnicas actuales de investigación documental. 3a. Ed. 2008. México: Trillas.

Coeller, R. (2012). Beyond the borders: Radicalized evangelical missionaries in Central America from the 1950 through the 1980. Submitted to the Faculty of the College of Arts and Sciences of American University in Partial Fulfillment of the requirements for the Degree of Doctor of Philosophy in History.

Comité de Lausana para la Evangelización mundial (1984). El Pacto de Lausana. Exposición y comentario por John Stott. Documentos periódicos de Lausana. No 3. Visión Mundial América Latina.

Comité Lausana El Salvador (2011). “Compromisos de Cuidad del Cabo. Sus desafíos para la iglesia evangélica en El Salvador". Delegación salvadoreña en el Congreso Lausana II, Cuidad del Cabo, Sudáfrica. San Salvador.

Dayton, E. (1980). That Everyone May Hear: Reaching the Unreached, 2nd. ed., Monrovia: MARC.

Dussel, E. (1992). Historia de la iglesia en América Latina: medio milenio de coloniaje y liberación (14921992). Mundo Negro-Esquila Misional. Madrid

Ellacuría, I. (2009). Cursos Universitarios: "El Sujeto de la historia”. 1a. Edición. UCA Editores. San Salvador.

Guerrero, M. (2010). El Pacto de Lausana (1974): una contextualización vista desde 2010. Integralidad. CEMAA. año 2, edición 8. Perú, Lima. 
Joshua Project. (2015) “Global Summary. An overview of the people groups of the world". Consultado el 3 de Mayo de 2016. https://joshuaproject.net/

Movimiento de Lausana. "El Compromiso de Ciudad del Cabo, Una Confesión de fe y un llamado a la Acción". Consultado el 3 de Mayo de 2016. http:// www.lausanne.org/es/contenido/compromiso-deciudad-del-cabo/compromiso

Movimiento de Lausana. "El Manifiesto de Manila: Llamado a toda la Iglesia a llevar todo el Evangelio a todo el mundo". Consultado el 3 de Mayo de 2016. http://www.lausanne.org/es/contenido/ manifiesto-de-manila/manifiesto

Padilla, C. R. (1985). "Evangelización y responsabilidad social, Wheaton '66 a Wheaton '83". Revista Iglesia y Misión, № 3, Sep. 1985, 83-90.

Padilla, R. (2006) "Bases Bíblicas de la Misión. Perspectivas latinoamericanas”. Nueva Creación.

Piedra, A. (2002). Evangelización protestante en América Latina. Análisis de las razones que justificaron y promovieron la expansión protestante.
Departamento de Comunicaciones del Consejo Latinoamericano de Iglesias. Ecuador, Quito.

S. Bevans y R. Schroeder (2009). Teología para la misión hoy. Constantes en contexto, Verbo Divino, Estella (Navarra).

Segura, H. (2006). "La Misión como liberación integral: Jesús, modelo sin igual". Kairos (38), EneroJunio, 23-38.

Sosa, C. (2007). “Aportes Misionológicos del protestantismo liberal en América Latina". Teología y Cultura. Año 4, Vol. 8.

Stanley, B. (2013), "Lausanne 1974: The Challenge of the Majority World to Northern-Hemisphere Evangelicalism". The Journal of Ecclesiastical History, vol. 64 , no. 3, 533-551.

"The Micah Declaration on Integral misión" ("La Declaración de la Red Miqueas sobre Misión Integral") en Tim Chester (ed.), Justice, Mercy and Humility: Integral Mission and the Poor (Justicia, Misericordia y Humildad: La Misión Integral y los Pobres). Carlisle, UK: Paternoster, 2002. 